\title{
A Flow Simulation in THE FOAMING PROCESS
}

\author{
Karel Fraňa ${ }^{1}$, Jörg Stiller ${ }^{2}$ and Iva Nová ${ }^{1}$ \\ ${ }^{1}$ Technical University of Liberec, Studentska 2, 46117 Liberec, Czech Republic \\ ${ }^{2}$ Technische Universität Dresden, Institut für Strömungsmechanik \\ 01062, Dresden, Germany
}

\begin{abstract}
This paper deals with unsteady three-dimensional numerical calculations of the two-phase flow problem represents a gas bubble formation in the liquid in the container with the specific size. For calculations, the Volume of Fluids approach is adopted to resolve the shape of bubbles and their dynamics. The liquid phase is a mixture of water-ethanol and the gas phase is considered as air. The problem is treated as isothermal. The study is still limited to the lower flow rates at which bubbles are created and rising separately without any interaction, merging etc. However, this particular problem still required finer mesh especially in the domain in which bubbles are formed. The current results showed that air bubbles have a form of the ellipsoid and after they reach the liquid surface, they are moving towards to the side walls along this liquid level. This fact is interesting from the view of the foaming process and for the other investigation of the bubble behaviour at this phase interface. The flow study is calculated parallel using compressible multi-phase flow solver.
\end{abstract}

\section{KEYWORDS}

Multi-phase flow simulations, Computational mesh, Parallel calculations \& Flow visualisation

\section{INTRODUCTION}

The numerical calculation of the multi-phase flow problem is an attractive approach to predict flow behaviours in technical applications. Generally, there are a lot for examples where it is possible to observe an interaction between liquid and gas phases in various equipment designs. If this flow feature of the multi-phase behaviour is known in advance due to numerical simulations, a design of real equipment can be improved. Consequently, it can save time and finally cost in the production phase of the prototype examination. Another problem, where the theoretical calculation of the multiphase flow problem can be applied, is a forming process used in the production of foam materials. There are several techniques to manufacture foams and the study is focused on the process in which the metal foams are directly fabricated by injecting a gas in molten metal alloys. To control this foaming process, the knowledge about bubble behaviour in the liquid is essential. If the temperature effects and solidification process should be considered as well, the problem becomes to be really complex [6]. Nowadays there are several ways how to calculate liquid-gas interface e.g. Volume of Fluids (VOF) approach, the immersed boundary method (IBM) etc. VOF method uses a function which denotes the fractional amount of particular fluid in at spatial location at time t. The advection term in equations is solved by pseudo-Lagrangian geometrical advection schemes in order to minimize numerical diffusion. This fact makes VOF [3] the most mass conservative among all multi-fluid interface tracking methods. This method was successfully applied for a problem of the bubbles generation and

David C. Wyld et al. (Eds): ICAITA, CDKP, SAI, NCO, CMC, SOFT, MLT, AdNLP - 2020 
rising bubbles in [2]. The two-phase flow over two types of step-poolspill was successfully investigated using two-phase schemes VOF and with various turbulence modelling in [5]. In contrast IBM uses non-body conformal structured Cartesian grids for simulating flow through complex geometries. The main advantages of IBM are simplicity in grid generation and discretization of the Navier-Stokes equations. So that it is easier to develop a code, less memory requirement to store the grid information and higher computational efficiency. In [1] an application of the IBM for a single rising bubble was discussed. This paper is focused on the investigation of rising gas bubbles in stagnant liquid. To obtain experimental results such as velocities, pressure, structure shape etc. will be generally difficult in aluminium alloys. The simulation study was carried out in the water-ethanol mixture with gas. Therefore obtained numerical results can be later validated by experiments. The paper is organised as follows: in chapter 2, the mathematical model, computational mesh and experimental background are introduced. Several founded results are presented and discussed in chapter 3. Chapter 4 contains conclusion to results and further scheduled work is briefly illuminated.

\section{Problem Formulation}

\subsection{Mathematical Model}

The essential point of the foaming process e.g. by the continuous gas injection is a doping of stabilization components into the melted alloys which cause a change of the material properties influencing further the flow features. This fact is a subject of the flow investigation. The gas injection is represented by air. The liquid phase is a mixture of water-ethanol which in fact represents aluminium alloys. The interface between gas and liquid fraction represents typically the two-phase flow problem. The three dimensional numerical simulation in the container is carried out in OpenFoam with the "CompressibleMultiPhaseInterFoam" Solver. The "heRhoThermo" model is used typically for the fixed mixture composition. For the gas fraction, the perfect gas equation is used in a form as follows,

$$
\rho=\frac{1}{R T} p,
$$

where $\rho$ is air density, $p$ pressure, $T$ temperature and $\mathrm{R}$ is gas constant. The compressibility of the gas phase is taken into account. There are several works that supposed the compressibility can be neglected under some specific circumstances e.g. in [12]. The fluid phase is considered to be perfect fluid with equation in (2),

$$
\rho=\frac{1}{R T} p+\rho_{0}
$$

where $\rho_{0}$ is the density at the temperature $T=0$. Material mixture properties are prescribed using the volume fraction function e.g. for the phase 1, which takes a form as

$$
\alpha_{1}=\frac{\Omega_{1}}{\Omega_{1}+\Omega_{2}+\Omega_{3}}
$$

where $\Omega_{1}, \Omega_{2}$ and $\Omega_{3}$ are volume fraction functions of the phase 1, 2 or 3, respectively. Similarly, the volume fraction function $\Omega_{2}$ can be formulated as in Eq.(3) or directly calculated using $\alpha_{2}=1$ $\alpha_{1}-\alpha_{3}$. A conservation law of mass for each component is defined as well. The equation of the mass conservation can be defined for 3 different phases taking a form as in Eq. (4),(5) and (6).

$$
\frac{\partial \alpha_{1} \rho_{1}}{\partial t}+\frac{\partial}{\partial x}\left(\alpha_{1} \rho_{1} u\right)+\frac{\partial}{\partial y}\left(\alpha_{1} \rho_{1} v\right)+\frac{\partial}{\partial_{z}}\left(\alpha_{1} \rho_{1} w\right)=0
$$




$$
\begin{aligned}
& \frac{\partial \alpha_{2} \rho_{2}}{\partial t}+\frac{\partial}{\partial_{x}}\left(\alpha_{2} \rho_{2} u\right)+\frac{\partial}{\partial_{y}}\left(\alpha_{2} \rho_{2} v\right)+\frac{\partial}{\partial_{z}}\left(\alpha_{2} \rho_{2} w\right)=0 \\
& \frac{\partial \alpha_{3} \rho_{3}}{\partial t}+\frac{\partial}{\partial_{x}}\left(\alpha_{3} \rho_{3} u\right)+\frac{\partial}{\partial_{y}}\left(\alpha_{3} \rho_{3} v\right)+\frac{\partial}{\partial_{z}}\left(\alpha_{3} \rho_{3} w\right)=0
\end{aligned}
$$

The velocity vector is given by $u, v$ and $w$ components. Density of each phase is denoted by $\rho_{1}, \rho_{2}$ or $\rho_{3}$. The equation of momentum takes a form as in Eq. (7), (8) and (9).

$$
\begin{gathered}
\frac{\partial}{\partial t}(\rho u)+\frac{\partial}{\partial x}\left(\rho u^{2}+p\right)+\frac{\partial}{\partial y}(\rho u v)+\frac{\partial}{\partial y}(\rho u w)=F_{x} \\
\frac{\partial}{\partial t}(\rho v)+\frac{\partial}{\partial x}(\rho u v)+\frac{\partial}{\partial y}\left(\rho v^{2}+p\right)+\frac{\partial}{\partial y}(\rho v w)=F_{y} \\
\frac{\partial}{\partial t}(\rho w)+\frac{\partial}{\partial x}(\rho w u)+\frac{\partial}{\partial y}(\rho v w)+\frac{\partial}{\partial y}\left(\rho v^{2}+p\right)=F_{z}
\end{gathered}
$$

$F_{x} . F_{y}$ and $F_{z}$ are components of the external forces e.g. surface tension forces or gravity force. The mixture density $\rho$ is used for all three equations (7), (8) and (9). The energy equation for temperature of the mixture is given in Eq. (10).

$$
\frac{\partial \rho T}{\partial t}+\frac{\partial}{\partial_{x}}(\rho u T)+\frac{\partial}{\partial_{y}}(\rho v T)+\frac{\partial}{\partial_{z}}(\rho w T)=w_{P}
$$

$\mathrm{W}_{\mathrm{p}}$ represents a pressure work which must be included if a compressible flow is expected. For definition of different flow state in regards to the bubble shape, several non-dimensional parameters are commonly used. The Eötvös number in Eq. (11) expresses the ration of the body force to the surface tension force,

$$
E o=\frac{\rho_{o} g D^{2}}{\sigma}
$$

Where $\rho_{0}$ is density of the continuous phase (in our case liquid mixture) and $\sigma$ is interfacial surface tension between gas and liquid phase. Parameter D is a characteristic length relating to the characteristic diameter of the formed bubble. The dynamics of bubble is influenced by the buoyancy force to the viscous force given by the Galilei number.

$$
G a=\frac{\rho_{0} g^{1 / 2} D^{3 / 2}}{\mu_{0}}
$$

In Eq. (12), the dynamics viscosity $\mu_{0}$ is related to continuous liquid phase. The Morton number can be calculated based on the Eo and Ga numbers as is denoted in Eq. (13).

$$
M o=\frac{E o^{3}}{G a^{4}}
$$

Relevant parameters Eo or Ga contains an information about bubble diameters, however, bubble dynamics is missing. In order to involve the bubble dynamics expresses by bubble velocities, bubble Renolds number (15) and Weber number (14) can be used.

$$
\begin{gathered}
W e=\frac{\rho u^{2} D}{\sigma} \\
R e=\frac{\rho u D}{\mu}
\end{gathered}
$$


Reynolds and Weber parameters can take into account the influence of the terminal velocity which can be found easily using numerical simulation. The terminal velocity can describe the unsteady bubble dynamics as well [11].

\subsection{Setting of Calculations}

For the iterative process of pressure quantity, a preconditioned conjugate gradient method is applied. Other quantities e.g. temperature or velocity etc. are iteratively calculated using Gauss Seidel schemes. Unsteady terms of mathematical models are treated using Euler scheme providing the accuracy of the first order. For the gradient operator, the Gauss linear scheme is applied; Laplacian operator is treated by Gauss linear uncorrected scheme and in the divergence operator of the most quantities Gauss linear scheme is adopted, however, due to specific instabilities in the calculation, for the velocities a Gauss upwind scheme had to be prescribed. Relaxation factors varied between 0.95 and 0.9 were applied for calculation of the velocity and pressure quantities.

\subsection{Computational Domain and Mesh}

The computational domain is determined by a real container with a size of $0.2 \mathrm{mx} 0.2 \mathrm{mx} 0.2 \mathrm{~m}$. The liquid phase level (water-ethanol mixture) is in the height of $0.12 \mathrm{~m}$. The rest of the computational domain represents the gas phase. The grid is refined at the place of the gas injection, at the container bottom, in the area of the rising bubble and at the interface of the gas and liquid phase. The computational mesh is shown in Figure 1.
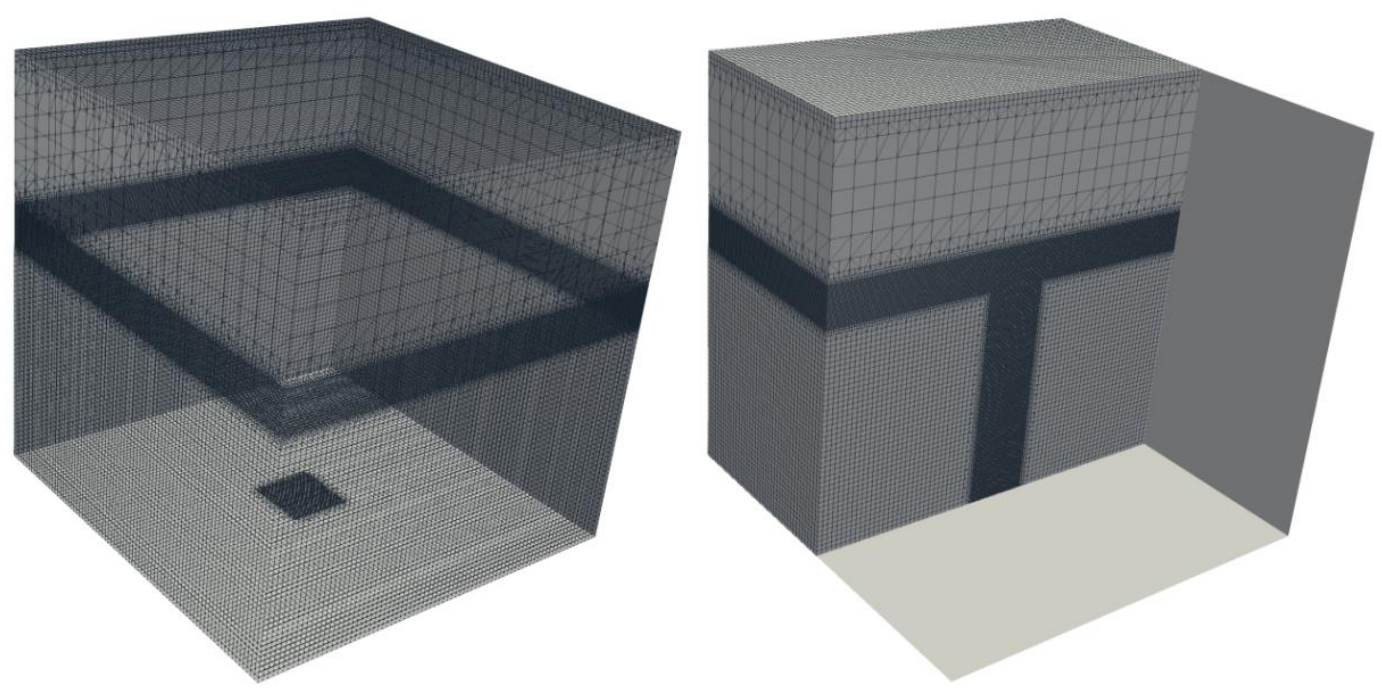

Figure 1. Computational mesh at the surface (left) and meshing inside (right) 


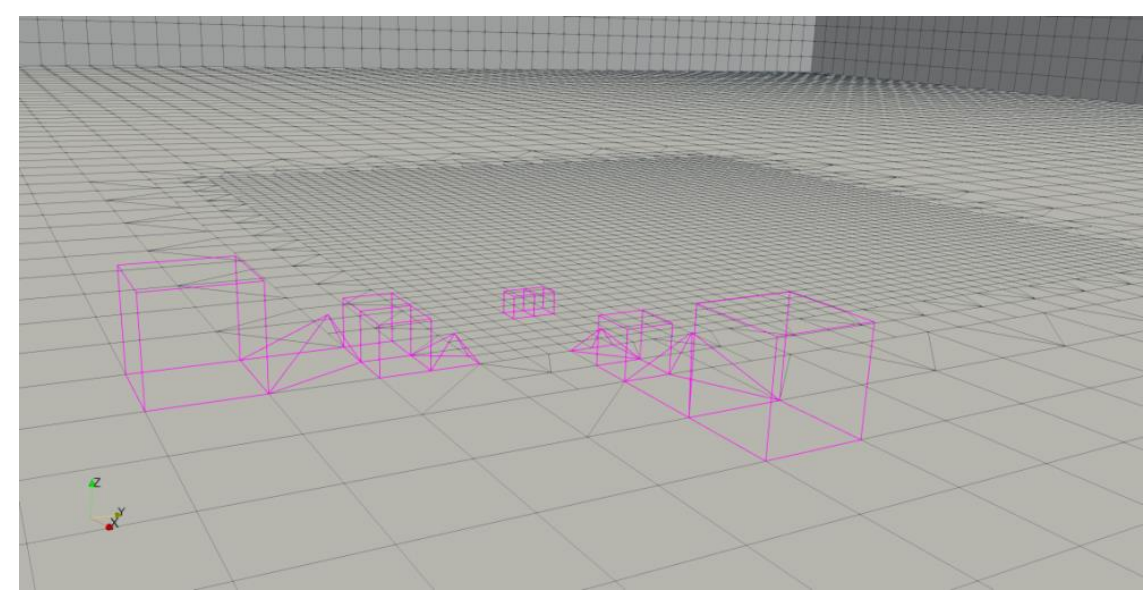

Figure 2. Details of the meshing in the area of the bubble generation

The mesh contains totally 4.1 mill cells, 4.01 mill. cells are hexahedral type, 0.78 mill. cells are polyhedral type. In the area, where the bubble creation and rising is expected, finer mesh resolution was applied in order to detect small changes of the bubble shape. Furthermore, the interaction between liquid and surrounding air (liquid level) is an objective of the investigation as well. Therefore, in this interface area, the fine mesh is adopted as well. Figure 2 shows the mesh surface at the bottom of the container with specific elements. In the layer representing the change of the cell size, different cell types are generated (hexahedral or polyhedral type). This mesh layer influences significantly the mesh quality e.g. the cell volume is $1 \times 10^{-6}$ for bigger hexahedral cell type, for smaller polyhedral cell type, this volume is of $2.44 \times 10^{-10}$. The maximal non-orthogonality is 25.23 and maximal skewness of cells is 0.33 . Both values are in tolerance recommended for finite volume methods.

\subsection{Foaming Process}

The experiment was carried out in the glass storage with dimensions of $30 \mathrm{~cm} \times 20 \mathrm{~cm} \times 20 \mathrm{~cm}$ (12 liters). At the bottom there is a nozzle connected to a flow meter and an air compressor. The surrounding temperature is $299 \mathrm{~K}$ and air flow rate is setup to be $100 \mathrm{~L} / \mathrm{h}$. In fact, experiments are performed at different molar ethanol fractions from 0 to 1 ; in order to illustrate how density and viscosity does change with molar fraction. The experiment started with 1 ethanol molar fraction $\left(\mathrm{X}_{\mathrm{e}}\right)$ and then water was being added gradually. In order to define changes, values of density and viscosity are measured simultaneously. 


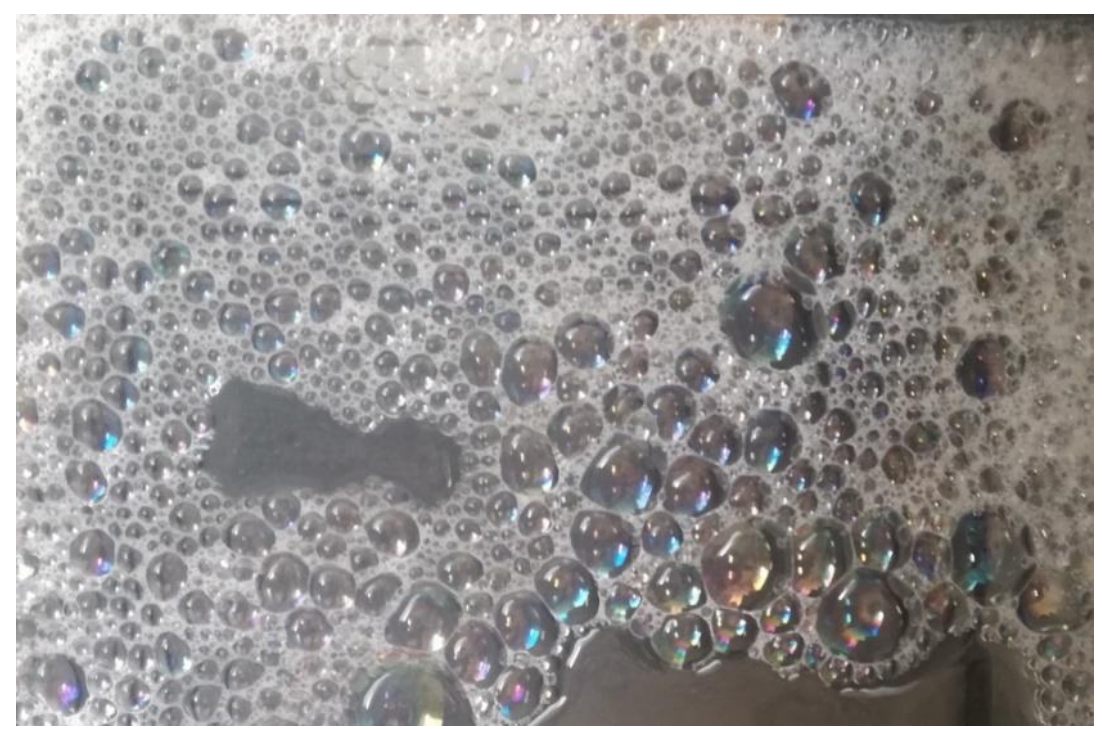

Figure 3. Bubbles accumulation after 4 minutes foaming at flow rate $1001 / \mathrm{h}$ and $\mathrm{Xe}=0.224$

Figure 3 shows typical foam created in the water-ethanol mixture with the molar ethanol fraction of $\mathrm{Xe}=0.224$. Experimentally, it was proved that foams are created only in the specific concentration of the Ethanol in the water leading to the specific material property mixture. Furthermore, the Eötvös number was determined to be in order of one.

\section{Results}

At the bottom of the container, the inlet air jet with the laminar velocity profile is prescribed. The maximal intensity of the inlet air velocity is $0.4 \mathrm{~m} / \mathrm{s}$, which correspond to the approx. $51 / \mathrm{h}$.

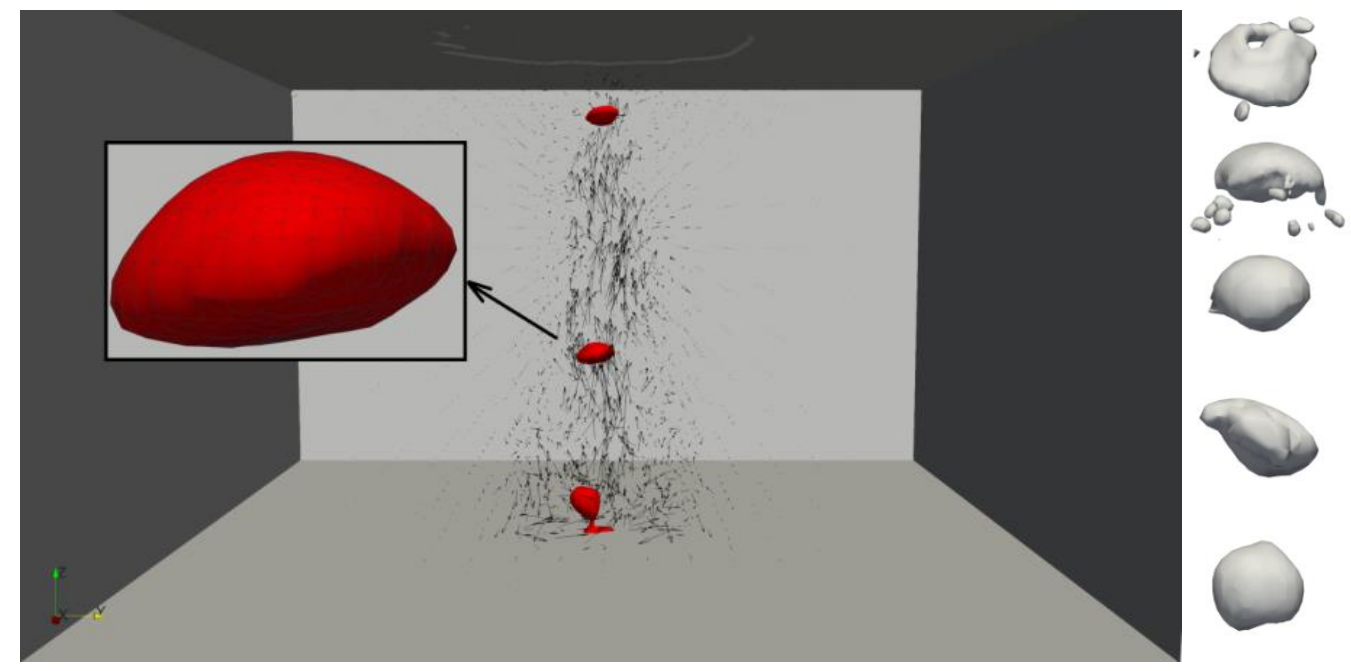

Figure 4. Instantaneous view on formed bubbles under liquid surface level (left) and detailed view on the bubble shapes (right)

This specific flow rate is relatively small which provides flow conditions to observe each bubble separately without merging or other form of the interactions. Figure 4 depicts the rising of bubbles with associated surrounding velocity field. The main flow inside of the container is connected with the dynamics of bubbles. According to e.g. [8], the three-dimensional numerical 
study identified an existence of different region of district bubble shapes depending e.g. on the Galilei and Eötvös numbers. In case illustrated in Fig. 4, the Galilei number varies between 400 and 800 in dependence on the distance from the gas jet onset. The Eötvös number is in a range between 2,2 to 5,4. For these values the so called IV region associated with spherical cap shape and breakup of bubbles [8]. Numerically, it was observed that how bubbles are rising up, they break up into the spherical caps and later, several smaller satellite bubbles are formed (see Fig. 4 right). Simultaneously, higher values of Galilei number indicate that the buoyancy effect of the bubble will be intensive than an effect of the viscosity. In [10], the relation between the Reynolds number and Weber and Morton numbers takes a form as in Eq. (16).

$$
R e=2.05 \mathrm{We}^{2 / 3} \mathrm{Mo}^{-1 / 5}
$$

In the numerical simulation carried out under following conditions defined by the Morton number of order $O\left(10^{-10}\right)$, the Eötvös number in order of $O(1)$, Weber number in order of $O(1)$ and Galilei number $O(100)$ was found that the bubble Reynolds number is approx. 500. This value of the bubble Reynolds number is in a relatively good match to the bubble Reynolds number calculated using formula in Eq. (16) to be approx. 600. In fact, this kind of the results validation is based on the velocity determination which is much more complicated than bubble diameter analysis for a Ga and Eo numbers. The same conclusion was found in [9] as well. Besides the bubble formation and further propagation, the main objective of the study is concerned on the foam creation.

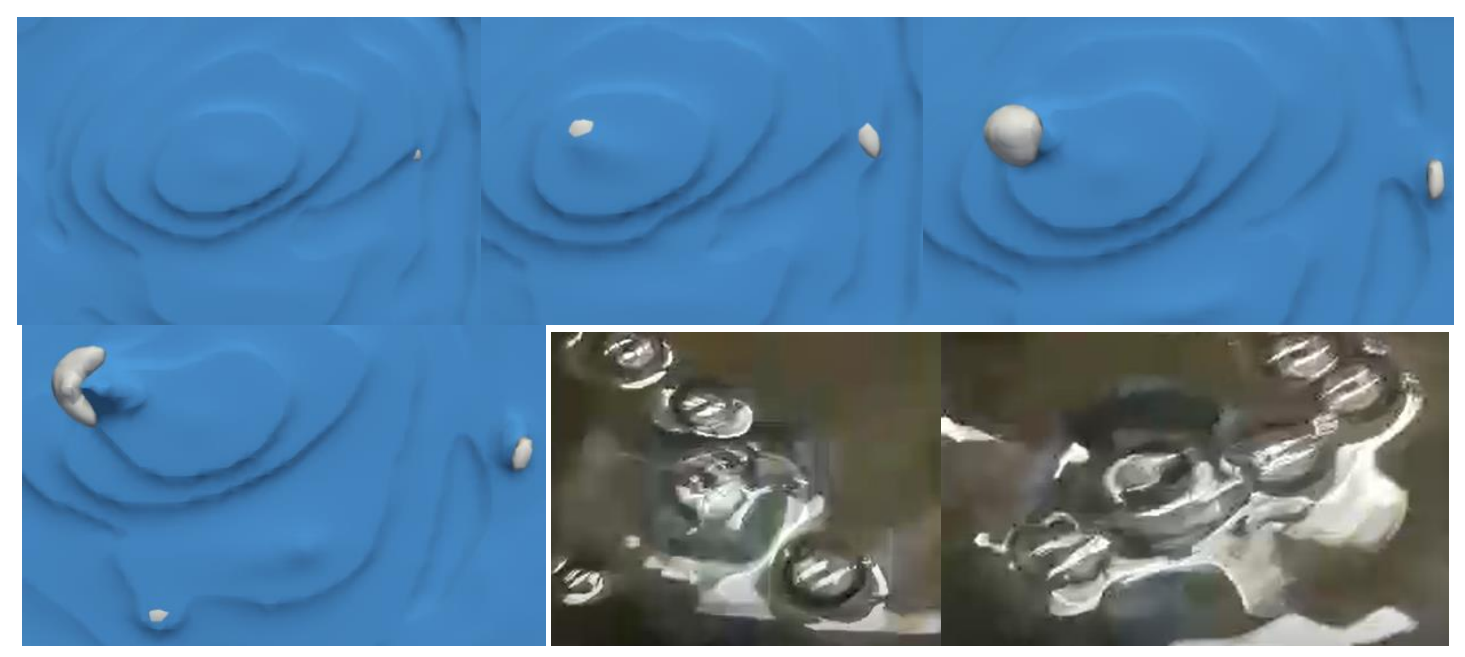

Figure 5. Calculated bubbles every $0.02 \mathrm{~s}$ captured at the liquid surface level (liquid surface level depicted by blue colour) vs. experiments

Figure 5 depicts the series of bubble formations on the liquid surface with the time step of $0.02 \mathrm{~s}$ and for lower intensity of foaming process. Bubbles after they reached the liquid surface start to move at the liquid surface towards wall or periodic conditions depending model configuration. The last two pictures in Fig. 5 shows experimental observation of bubbles formed at the liquid surface. Similarly to numerical results, bubbles are moving separately in same direction, however, no bubble break up is observed in contrast to numerics. 


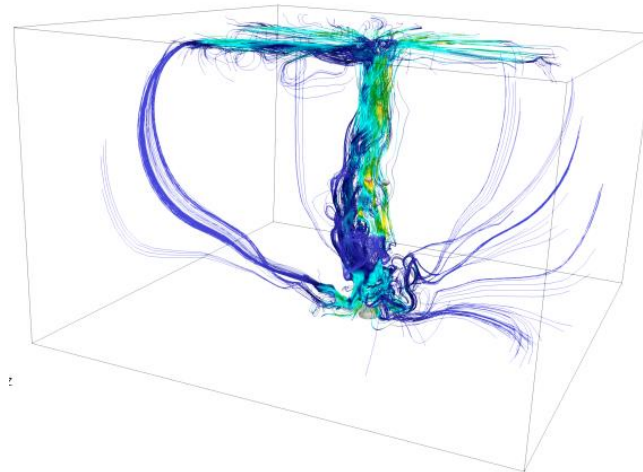

(a)

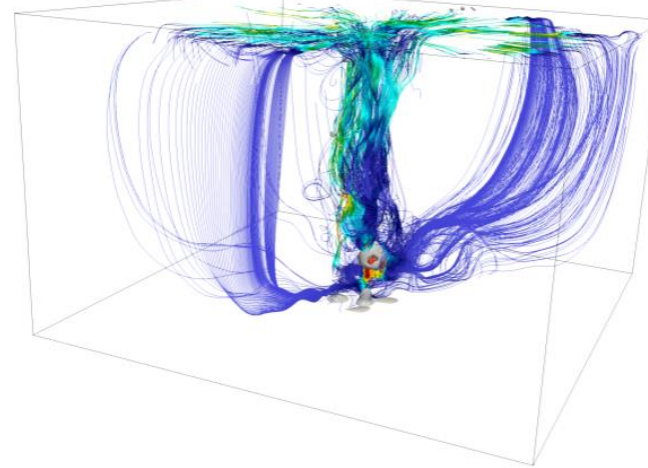

(c)

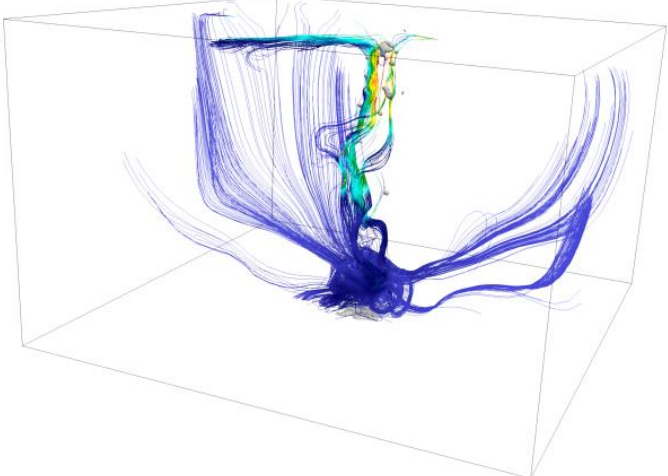

(b)

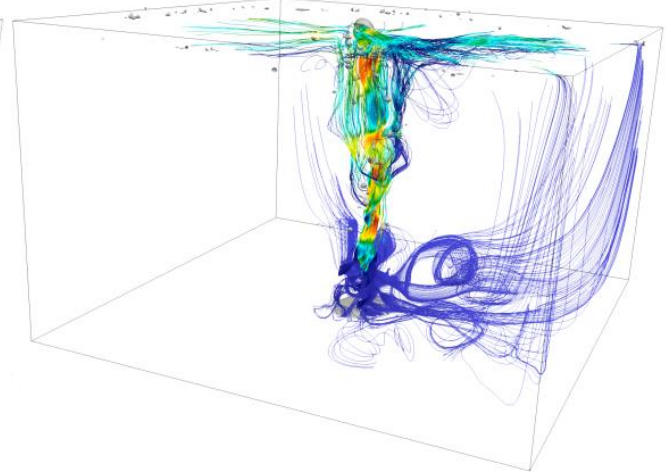

(d)

Figure 6. Path lines with velocity depictions for periodic boundaries at the vertical boundary (a) in time $2.91 \mathrm{~s}$ and (b) $3.45 \mathrm{~s}$ and for walls at the vertical boundary (c) $2.91 \mathrm{~s}$ and (d) $3.45 \mathrm{~s}$

Therefore, the detailed study is required to simulate bubble behaviour just at the liquid surface, where interface between gas and surrounding liquid phase and again gas phase is defined by very small thickness. This fact can be reason that bubbles cannot keep form and start to break up or dissolve. This so called soap film behaviour differences phases can be expressed by the balance between forces on the film in terms of the pressure difference [7] given by Eq. (17).

$$
\Delta p=\frac{8 \sigma}{D}
$$

The foaming process and resulting foams are influenced by the side walls. Figure 6 shows path lines in the investigated domain for periodic or wall side boundary conditions. Colours indicate the intensity of the velocity, where red colour corresponds to the $0.4 \mathrm{~m} / \mathrm{s}$ which is maximal velocity in the core of the air jet used in the air injection at the bottom. The flow at the liquid surface level is responsible for the bubble transport from the centre of container towards side wall. The liquid surface velocity is asymmetrical any boundary conditions and varies on the time. In case of wall conditions, the entire domain is influenced by gas jet, however, in case of periodic conditions; the upper part of the container is less influenced by the gas jet flow. 


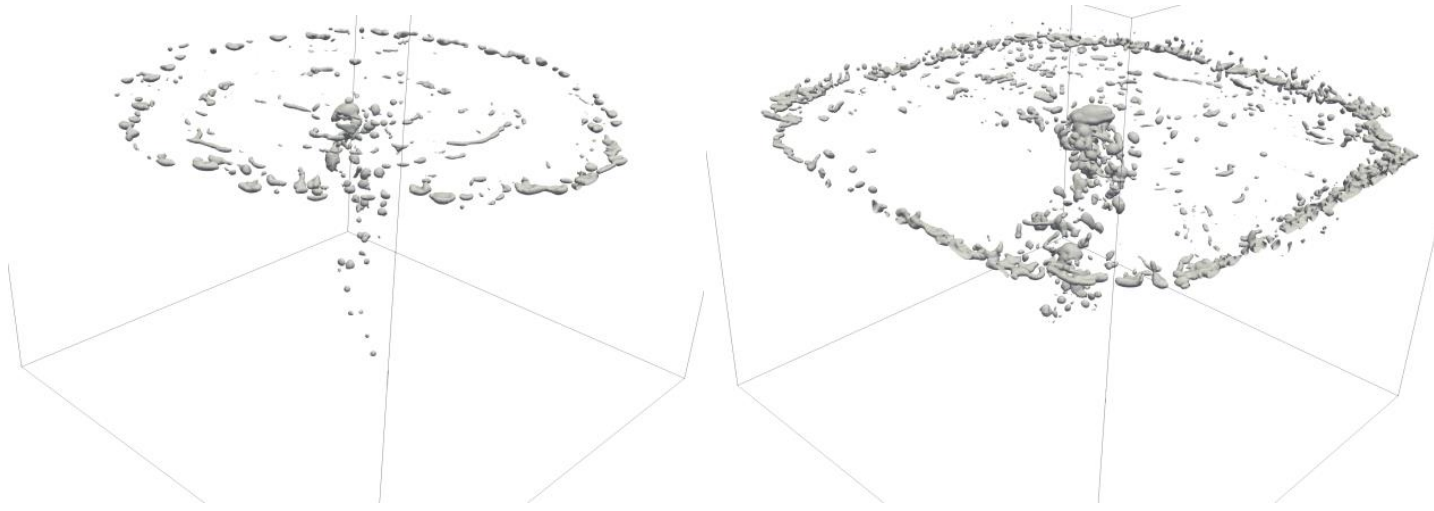

Figure 7. Bubble structures at the liquid free surface for periodic side vertical boundary (left) and wall type vertical boundary (right)

Figure 7 shows the air structures of bubbles which are rising upwards and simultaneously bubble structures captured at the free surface of the liquid for periodic or wall type vertical boundaries. It is evident that higher accumulation of the gas phase is concentrated if the wall type conditions are prescribed at the vertical walls. To achieve so high concentration of bubbles at the liquid free surface, a long time simulation is required particularly more than $2 \mathrm{~s}$ of calculation. The time step of the calculation is limited to the maximal courant number of 0.95 however, in fact, this value is varying around 0.6. The size of the time steps depends on the maximal velocity appeared in the velocity field. It was detected in simulations the significant velocity oscillation mostly by the insipience of the bubbles at the bottom. These higher velocities reduce for a particular time period the size of the time step used for calculations. In fact the time step is varying from $5 \times 10^{-5} \mathrm{~s}$ to $2 \times 10^{-4} \mathrm{~s}$.

\section{Conclusions}

The two-phase flow problem of the bubble formation on the created foam at the liquid free surface was studied numerically using the compressible multi-phase flow solver based on the Volume of Fluids approach. For fluid phase, the mixture of ethanol-water with specific molar fraction of the ethanol was considered to replace real aluminum alloys. The temperature effect and solidification are completely neglected. The main objective of the study is the bubble behavior in this isothermal homogeneous liquid mixture and the foaming process. It was found that the compressible multi-phase flow solver was able to predict the bubble formation and its dynamics. Results were compared with other experimental and numerical results. Furthermore, using appropriate structure visualization, the original bubbles are possible to still observe later on the liquid free surface where they stay to be floating. However, the strong gas phase dissipation appeared maybe because of the insufficient mesh resolution. This part of the flow at the free surface level needs to be investigating further. On the other hand, different requirements for the mesh resolution causes non-homogenous mesh in which the mesh quality must be carefully check it. In perspectives, the simulation will continue to find main differences between mixture of water-ethanol and poor water. Simultaneously, the impact of the side boundaries on the foams will be going on. In this study, it was found that vertical boundaries can influence the foaming process and in case of the walls at the vertical boundaries, higher concentration of gas phase fraction was observed. 


\section{ACKNOWLEDGEMENTS}

This work was supported by the Ministry of Education, Youth and Sports of the Czech Republic and the European Union - European Structural and Investment Funds in the frames of Operational Programme Research Development and Education - project Hybrid Materials for Hierarchical Structures (HyHi, Reg. No. CZ.02.1.01/0.0/16-019/0000843).

\section{REFERENCES}

[1] Sebastian Aland, Stephan Schwarz, Jochen Fröhlich \& Axel Voigt, (2013) "Modeling and numerical approximations for bubbles in liquid metal“, Eur. Phys. J. Special Topics 220, 185 -194.

[2] Karel Fraňa, Shehab Attia, Jörg Stiller, (2019) "A Bubble Formation in the Two-Phase System", In: Rodrigues J. et al. (eds) Computational Science - ICCS 2019. Lecture Notes in Computer Science, vol 11539. Springer.

[3] C.W. Hirt \& B.D. Nichols, (1981) "Volume of fluid (VOF) method for the dynamics of free boundaries“, J. Comput. Phys. 9(1), 221-225.

[4] Karel Fraňa \& Iva Nová, (2018) "A interface formation in two-phase flow problems", International Journal of Mechanics, 12, pp. 102-108.

[5] Y. Yang, S. Wang \& C. Wen, (2017) "Gas-liquid two-phase flows in double inlet cyclones for natural gas separation", Cogent Engineering 4(1).

[6] A. Hasečić, S. Muzaferija \& I. Demirdžić, (2020) "Finite volume method for multiphase flows with radiation and phase change", International Journal of Thermal Sciences, Vol. 149, 106201.

[7] E. Rio \& AL. Biance, (2014) "Thermodynamic and mechanical timescales involved in foam film rupture and liquid foam coalescence", Chemphyschem, Dec;15(17), pp. 3692-3707. doi:10.1002/cphc.201402195.

[8] D.M. Sharaf, A.R. Premlata, Manoj Kumar Tripathi, Dadarinath Karri \& Kirti Chandra Sahu, (2018) "Shapes and paths of an air bubble rising in quiescent liquids", Physics of Fluids 29, 122104, https://doi.org/10.1063/1.5006726.

[9] Dominique Legendre, Roberto Zenit, \& J. Rodrigo Velez-Cordero, (2012) "On the deformation of gas bubbles in liquids", Physics of Fluids 24, 043303, https://doi.org/10.1063/1.4705527.

[10] Marie Rastello, Jean-Louis Marié \& Michel Lance, (2010). "Drag and lift forces on clean spherical air bubbles in a rotating flow", J. Fluid Mech. 682, 434.

[11] Mitsuhiro Ohta \& Mark Sussman, (2012) „The buoyancy-driven motion of a single skirted bubble or drop rising through a viscous liquid“, Physics of Fluids 24, 112101, https://doi.org/10.1063/1.4765669.

[12] M.K. Tripathi, K.C. Sahu \& R. Govindarajan, (2015) "Dynamics of an initially spherical bubble rising in quiescent liquid" Nature communications, 6, 6268. 


\section{AUTHORS}

Karel Frana Professor at the Technical university of Liberec, Faculty of Mechanical Engineering, research field: numerical simulations, multiphase flow problems, energy efficiency; More than 20 publications in International Journals, more than 50 conference papers, more than 10 contributions in books.

Jörg Stiller Professor at Technische Universität Dresden, Institut für Strömungsmechanik Reseach field: computational fluid dynamics, multi-level methods with higher orders, turbulent flows, aerodynamic Recently: more than 60 papers in journals or conferences.

Iva Nová Senior Professor at the Technical university of Liberec, Faculty of Mechanical Engineering, research field: metal structures, foaming processes, experimental investigation, metal solidifications; More than 20 publications in International Journals, more than 50 conference papers, more than 10 contributions in books.
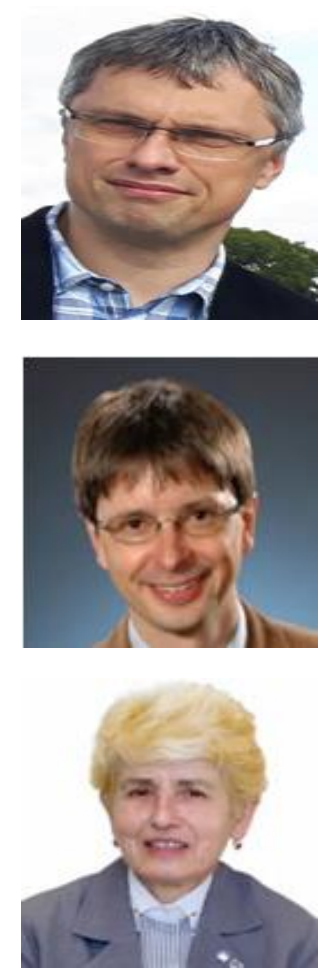

(C) 2020 By AIRCC Publishing Corporation. This article is published under the Creative Commons Attribution (CC BY) license. 